\title{
Autowaves in the moving excitable media
}

\author{
V.A.Davydov ${ }^{1}$, N.V.Davydov ${ }^{1}$, V.G.Morozov ${ }^{1}$, \\ M.N.Stolyarov ${ }^{2}$, T.Yamaguchi ${ }^{3}$ \\ 1 Moscow State Institute of Radioengineering, Electronics and Automation, \\ 117454, Vernadsky Prospect 78, Moscow, Russia \\ 2 Department of theoretical physics, Physical Institute of Russian Academy \\ of Sciences, 117924, Leninsky Prospect 53, Moscow, Russia \\ 3 National Institute of Advanced Industrial Science and Technology (AIST), \\ Higashi, 1-1-1, Tsukuba, Ibaraki 305-8565, Japan
}

Received April 5, 2004

Within the framework of kinematic theory of autowaves we suggest a method for analytic description of stationary autowave structures appearing at the boundary between the moving and fixed excitable media. The front breakdown phenomenon is predicted for such structures. Autowave refraction and, particulary, one-side "total reflection" at the boundary is considered. The obtained analytical results are confirmed by computer simulations. Prospects of the proposed method for further studies of autowave dynamics in the moving excitable media are discussed.

Key words: nonlinear dynamics and nonlinear dynamical systems, reaction-diffusion media, autowave refraction

PACS: $05.50 .+q, 05.70 . \operatorname{Ln}, 82.40 .-g, 87.10 .+e$

\section{Introduction}

There are many physical, chemical, and biological systems which may be regarded as excitable media consisting of locally connected active elements capable of forming pulses [1]. The most intriguing property of excitable media is the existence of autowaves, i.e., nonlinear spatiotemporal structures propagating through the medium. Autowave patterns is one of the most dramatic examples of self-organization in macroscopic systems $[1,2]$.

Over many years, most attention has been concentrated on the study of autowave processes in plane homogeneous media. More recently, however, the trend has been toward the study of autowaves in anisotropic, curved, inhomogeneous, and non-stationary excitable media. This range of problems is of great importance in practice. Using feed-back techniques, such as enhancing catalytic efficiencies, fo- 
cused laser light, imposing gradients of excitability, etc., it is possible to control autowave propagation. Another promising way of controlling the autowave patterns is based on the fact that, in the moving excitable media, gradients of concentration of different species, velocity gradients, and temperature gradients can dramatically modify the behavior of autowaves. Our aim in the present paper is to consider some new effects in the moving excitable media.

Distributed excitable systems are usually described by a set of reaction-diffusion equations

$$
\frac{\partial \boldsymbol{U}}{\partial t}=\boldsymbol{F}(\boldsymbol{U})+\hat{D} \cdot \Delta \boldsymbol{U}
$$

where $\boldsymbol{U}(\boldsymbol{r}, t)$ is a vector whose components $U_{n}$ give spatial concentrations of reacting species. The source term $\boldsymbol{F}$ specifies the local reactions in small volumes of the medium, and $\hat{D}$ is the matrix of transport (diffusion or thermoconductivity) coefficients. Equations (1.1) may be viewed as a "microscopic" model of excitable media.

Unfortunately, general methods for the analytic solution of equations (1.1) are not available. Therefore, these equations serve as a basis for numerical and other approximate methods. In many realistic reaction-diffusion systems, the local curvature $k$ of wave fronts is so small that the curvature radius $R=1 / k$ is much larger than the wave width $a$. In such cases a wave can be represented by a smooth infinitely thin oriented curve. This approximation is fundamental for the so-called kinematic approach (see, e.g., [3,4]) which is successfully employed in the studies of large-scale phenomena in 2D and 3D excitable media. In the region of its applicability the kinematic approach is proved to give results which are in good agreement with those obtained by numerical calculations of reaction-diffusion equations (1.1). It should be noted that the kinematic approach offers some advantages over direct numerical schemes. First, in many cases the results can be obtained in analytic form. This provides new and interesting insights into the behavior of autowave structures. Second, qualitative predictions of the kinematic approach are "universal" in a sense that they refer to any reaction-diffusion excitable medium.

Autowaves in the moving excitable media, essentially under shear flow, were studied by many authors (for references see, e.g., recent papers $[5,6]$ ). The most common theoretical approach in all these studies is based on numerical solution of reaction-diffusion equations (1.1) with some specific expressions for generic reactivity functions in the source term $\boldsymbol{F}$. Within the kinematic approach, the problem of autowave structures in the moving media was posted for the first time in [7]. In the present paper the method outlined in [7] will be used to describe stationary autowave structures appearing at a boundary between the moving and fixed 2D excitable media.

The paper is organized as follows. Section 2 reviews stationary autowave structures in a homogeneous excitable medium. Section 3 turns to stationary structures appearing at a boundary between the moving and fixed media with tangential velocity discontinuity. We show that such structures can be represented in terms of the stationary excitation fronts in an infinite homogeneous medium. Critical properties 
of autowave structures at the boundary are discussed. Section 4 deals with autowave refraction at the boundary between semi-infinite moving and fixed media. Section 5 presents the results of computer simulations confirming theoretical predictions. Section 6 concludes with remarks on further applications of the kinematic method to the studies of autowave structures in excitable media with penetrable boundaries.

\section{Non-spiral autowave structures in homogeneous media}

We start with basic kinematic equations that are necessary for our discussion.

As already noted, the kinematic description reduces an autowave structure to an infinitely thin excitation front. At any time $t$ this front can be specified by its natural equation $k(l, t)$ which gives the curvature $k$ as a function of the path length $l$ measured from a certain point on the front. Assuming that each small segment of the front moves in its normal direction with some local velocity $V(l, t)$, simple considerations lead to the evolution equation $[3,4]$

$$
\frac{\partial k}{\partial t}+\frac{\partial k}{\partial l}\left(\int_{0}^{l} k V \mathrm{~d} \xi+C\right)+k^{2} V+\frac{\partial^{2} V}{\partial l^{2}}=0
$$

where $C$ is the growth velocity of free tips of the front. The kinematic approach supposes that $V$ and $C$ depend on $l$ through the local curvature. For $k$ considerably smaller than the inverse width of the excitation pulse, this dependence can be taken as linear. The relation for the local velocity has the form

$$
V=V_{0}-D k
$$

where $V_{0}$ is the velocity of the plane front, and $D$ is the diffusion coefficient. Equation (2.2) is known in the literature as the eikonal equation [8].

In this paper we will be interested in steady-state autowave structures moving with constant shape and velocity. In such cases $\partial k / \partial t=0$ and $C=0$. Then, after one integration over $l$, equation (2.1) becomes

$$
k \int_{0}^{l} k V \mathrm{~d} \xi+\frac{\mathrm{d} V}{\mathrm{~d} l}=0
$$

Generally speaking, the right-hand side of this equation is equal to an integration constant $\omega$ which has the meaning of the angular speed of rotation of the front [4]. In what follows we will consider only non-spiral autowave structures, so that we put $\omega=0$.

Before proceeding to a study of the moving excitable media with boundaries we shall touch briefly on stationary autowave structures in an infinite homogeneous medium. In this case equation (2.3) is exactly integrable if the local velocity is given by $(2.2)$. The solutions were fully considered in $[9,10]$. Here we run through the structures available for different values of the curvature $k(0)$ at the point $l=0$. 


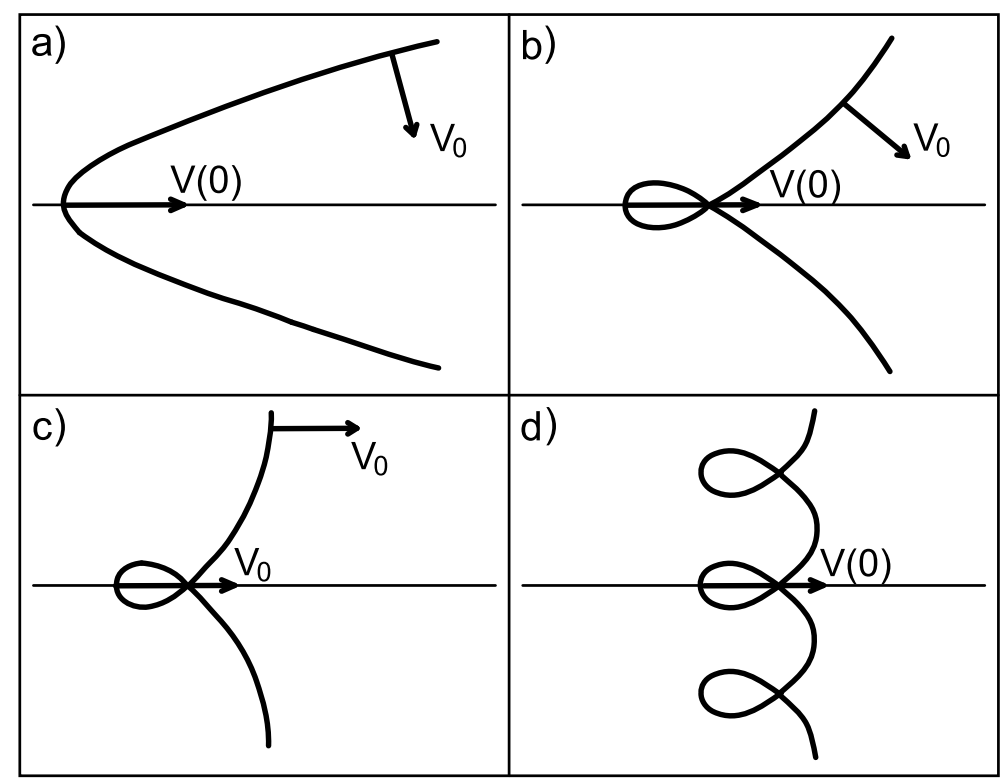

Figure 1. Non-spiral stationary autowave structures in a homogeneous excitable media: a) $V$-shaped pattern; b) self-intersecting loop; c) separatrix; d) multi-loop.

First we note that equation (2.3) has two trivial solutions: $k=0$ (a plane front) and $k=V_{0} / D$ (a stationary circle). Let us list the non-trivial steady-state configurations:

(i) For $k(0)<0$, equation $(2.3)$ describes the so-called $V$-shaped patterns (figure 1a). The explicit expression for $k(l)$ is given by

$$
k(l)=-\frac{1}{D} \frac{V^{2}(0)-V_{0}^{2}}{V_{0}+V(0) \cosh \left[\left(\sqrt{V^{2}(0)-V_{0}^{2}} / D\right) l\right]},
$$

where $V(0)=V_{0}-D k(0)$. It is notable that the stable $\mathrm{V}$-shaped patterns in excitable media were first predicted within the kinematic approach [9]. Shortly thereafter they were observed experimentally in the Belousov-Zhabotinsky (BZ) reaction [11].

(ii) For $k(0)>2 V_{0} / D$, the stationary solution of equation (2.3) is the self-intersecting loop (figure 1b):

$$
k(l)=\frac{1}{D} \frac{V^{2}(0)-V_{0}^{2}}{V(0) \cosh \left[\left(\sqrt{V^{2}(0)-V_{0}^{2}} / D\right) l\right]-V_{0}} .
$$

(iii) In the case that $k(0)=2 V_{0} / D$, equation (2.3) gives

$$
k(l)=\frac{2 V_{0} / D}{1+\left(V_{0} l / D\right)^{2}} .
$$

Following [10], we shall call this configuration (figure 1c) the separatrix. 
(iv) Finally, for $0<k(0)<V_{0} / D$ and $V_{0} / D<k(0)<2 V_{0} / D$, the solution of equation (2.3),

$$
k(l)=-\frac{1}{D} \frac{V^{2}(0)-V_{0}^{2}}{V_{0}+V(0) \cos \left[\left(\sqrt{V^{2}(0)-V_{0}^{2}} / D\right) l\right]},
$$

represents the multi-loop shown in figure $1 \mathrm{~d}$.

A few comments should be made here about the above-listed autowave structures. Using the method developed in [4], it can be shown that all these structures are stable in a sense that their profiles are recovered after small perturbations. Note, however, that structures with self-crossing cannot propagate in homogeneous infinitely extended media because autowave pulses annihilate after collision. This effect is not described by the kinematic equation (2.1). Thus, the only stable non-spiral autowave structures in infinitely extended media are plane fronts and V-shaped patterns. Nevertheless, in the next section we will show that non-self-crossing branches of the other structures can exist near a boundary between the moving and fixed excitable media.

\section{Stationary autowave structures at a boundary between the moving and fixed media}

In order to gain insight into characteristic properties of autowave structures in the moving media, we will consider a homogeneous medium in a strip of width $2 h$ which is infinitely extended along the $X$-axis. Zero-flux conditions will be used for both boundaries of the strip (i. e., there is no diffusion of the reacting substances through the boundaries). Suppose also that the strip was cut into two strips each of width $h$, and the upper one moves with velocity $\boldsymbol{w}$ along the $X$-axis (see figure 2 ). This system with the tangential velocity discontinuity can easily be realized experimentally and, besides, it may serve as a basis for the study of autowave structures in more complicated systems, say, in excitable media with shear flow.

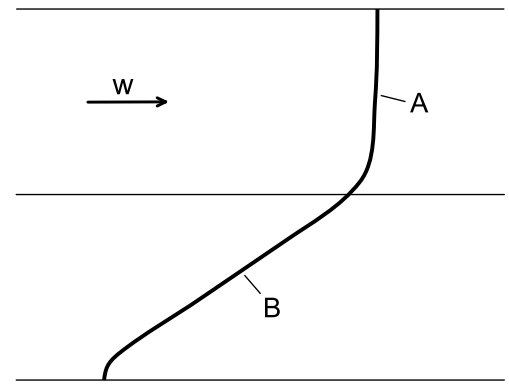

Figure 2. A stationary autowave structure at the boundary between moving and fixed excitable media. (A) is a branch of the separatrix; (B) is a branch of the $V$-shaped pattern. The wave moves along the relative velocity of the media $\boldsymbol{w}$. 
Our aim now is to search for steady-state autowave structures which can be generated in the system described above. It is clear that an excitation front propagating along the $X$-axis with constant velocity must be constructed from branches of the stationary solutions of equation (2.3) listed in the previous section. This alleviates the problem.

We shall restrict our consideration to the case where the width of the strip $h$ is very much larger than the characteristic length $l_{0}=D / V_{0}$ which determines the size of the separatrix loop, equation (2.6). Taking the values $D=2 \cdot 10^{-5} \mathrm{~cm}^{2} / \mathrm{c}$ and $V_{0}=3 \mathrm{~mm} / \mathrm{min}$, which are typical of the BZ reaction, we obtain $l_{0}=0.04 \mathrm{~mm}$. This estimate shows that the case where $h \approx l_{0}$ is very unlikely to be experimentally realizable.

Since the upper and lower boundaries of the medium are assumed to be impervious to diffusion, an autowave front must be orthogonal to them (see, e.g., [4]). Then it can be easily verified that in the case $h \gg l_{0}$ the only steady-state autowave which can propagate along the $X$-axis is the solution of equation (2.3) constructed from the branch of the separatrix (in the moving medium) and the "half" of the $V$-shaped pattern (in the fixed medium). This solution is pictured in figure 2.

Note that the excitation front shown in figure 2 moves as a whole along the $X$-axis with velocity $V_{0}+w$ because its part in the upper strip is the branch of the separatrix (see figure 1c) and $h \gg l_{0}$. Taking $l=0$ at the tip of the structure on the lower boundary of the fixed medium, we find that for the $V$-shaped pattern $V(0)=V_{0}+w$. This condition, together with equation (2.2), determines uniquely the excitation front in the fixed medium. The essential point is that the negative curvature of almost all front line of a $V$-shaped pattern, equation (2.4), is close to zero and only near the vertex it becomes very large (see, e.g., $[9,11])$. Thus, due to the condition $h \gg l_{0}$, the front of the $V$-pattern near the boundary between the media in figure 2 is nearly a straight line. The angle $\beta$ between this front and the boundary can be easily determined by the parameter $V(0)$ as [11]

$$
\sin \beta=V_{0} / V(0)=V_{0} /\left(V_{0}+w\right) .
$$

The curvature $k_{\mathrm{b}}$ of the front in the moving medium near the boundary differs from zero and, as shown below, essentially depends on the velocity $w$. To calculate $k_{\mathrm{b}}$, we note that the sewing condition requires that the velocities of the $V$-pattern and the separatrix along the $X$-axis should be the same at the boundary between the media. Let $V_{\mathrm{b}}$ be the corresponding normal velocity of the front in the upper strip (in the co-moving frame of reference). Then, taking into account that the asymptotic normal velocity of the $V$-pattern must be equal to the velocity of the plane front, the sewing condition can be written as

$$
V_{0} / \sin \beta=V_{\mathrm{b}} / \sin \beta+w .
$$

Since $V_{\mathrm{b}}$ and $k_{\mathrm{b}}$ satisfy the eikonal equation (2.2), from (3.1) and (3.2) it follows that

$$
k_{\mathrm{b}}=\frac{1}{D} \frac{V_{0} w}{V_{0}+w}
$$


This result, together with equation $(2.6)$, determines the value $l=l_{\mathrm{b}}$ on the boundary and, consequently, the shape of the front in the moving medium.

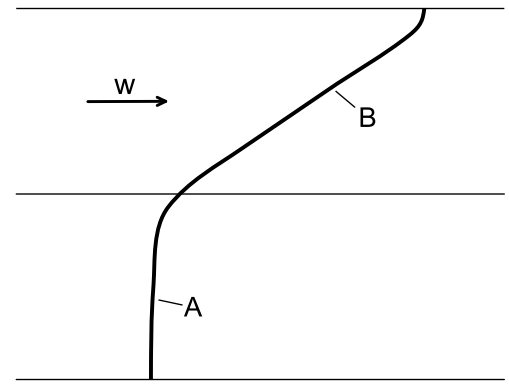

Figure 3. The same as in figure 2 , but the wave moves in the direction opposite to the relative velocity of the media $\boldsymbol{w}$.

Equation (3.3) shows that the curvature $k_{\mathrm{b}}$ of the front in the moving media near the boundary is a monotonically increasing function of $w$. This leads to a remarkable effect which does not occur when linear waves (for instance, electromagnetic waves) are refracted on a boundary between the moving and fixed media. It is known that, for autowave fronts, there exists a critical curvature $k^{*}\left(k^{*}<V_{0} / D\right)$, above which stable propagation becomes impossible $[12,13]$. Turning to the situation shown in figure 2, we may state that for some critical velocity $w^{*}$ the steady-state structure described above becomes unstable. The value of $w^{*}$ follows from (3.3) if we set there $k_{\mathrm{b}}=k^{*}$ :

$$
w^{*}=\frac{V_{0}}{V_{0} /\left(D k^{*}\right)-1} .
$$

For $w>w^{*}$, the excitation front will be broken at the boundary, so that the autowaves in the moving and fixed media will propagate independently of one another.

We have considered the case where the autowave structure moves in the direction of the relative velocity $\boldsymbol{w}$ of the media. If the excitation front moves in the opposite direction, then the only possible steady-state configuration is constructed from the "half" of a $V$-shaped pattern in the moving medium and the branch of the separatrix in the fixed medium (see figure 3). Following the same line of reasoning as before, it is easy to derive the stationary front shape and the critical velocity $w^{*}$. It is interesting to note that the corresponding expressions coincide with those cited above.

\section{Refraction of autowaves}

In the limit that $h \rightarrow \infty$ in figure 2 , we deal with a plane boundary between the moving and fixed semi-infinite excitable media. Stationary solutions of equation (2.3) will now describe refraction of autowaves at the boundary.

Clearly the structures considered in the previous section (figure 2 and figure 3 ) are stable as before if the curvature (3.3) does not exceed the critical value $k^{*}$. The only new point is that now the vertex of the $V$-pattern goes to infinity and the corresponding branch of the steady-state structure becomes a real plane front. 
Note, however, that in the case of semi-infinite media new stable stationary structures are possible. They are constructed from the plane front and the non-selfcrossing branch of the loop (see figure 4).

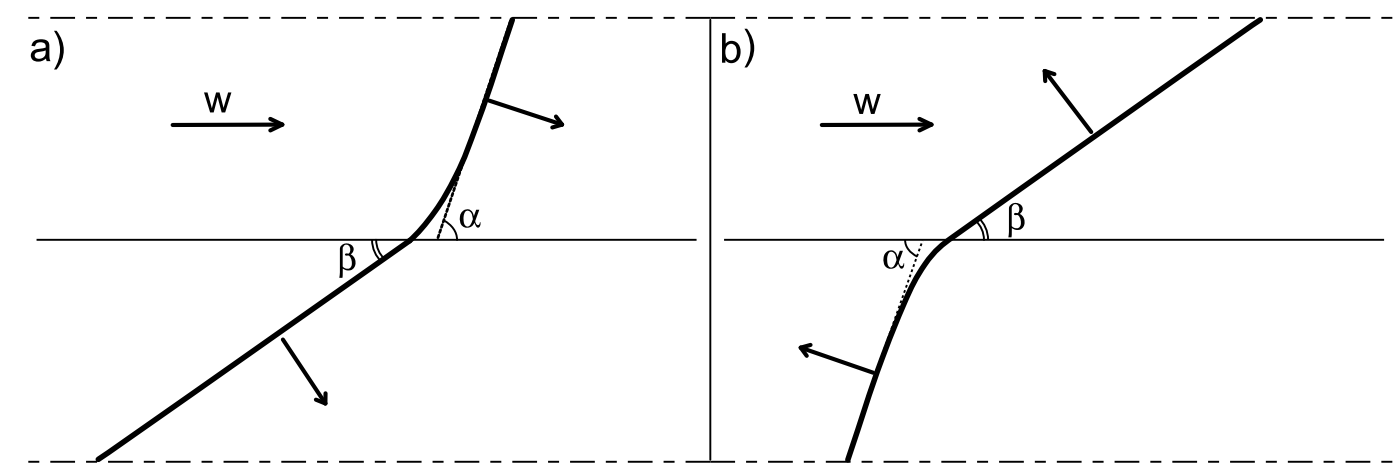

Figure 4. Autowave refraction at the boundary between the moving and fixed excitable media.

Using the natural equation for the loop, equation (2.5), and recalling the arguments from the previous section, it is an easy matter to give an exhaustive analysis of autowave refraction. For the structure shown in figure $4 \mathrm{a}$, as an example, we obtain the refraction law

$$
\sin \beta=\frac{\sin \alpha}{1+w \sin \alpha / V_{0}},
$$

where $\alpha$ is the angle between the boundary and the asymptote of the loop front in the moving medium ("incidence angle"), while $\beta$ is the angle between the boundary and the plane front in the fixed medium ("angle of refraction"). The curvature of the loop branch at the boundary is derived from equations (2.5) and (4.1):

$$
k_{\mathrm{b}}=\frac{1}{D} \frac{V_{0} w \sin \alpha}{V_{0}+w \sin \alpha} .
$$

Finally, for a given $\alpha$, the continuous excitation front shown in figure 4a breaks at the boundary when the relative velocity of the media $w$ exceeds the critical value

$$
w^{*}(\alpha)=\frac{w^{*}}{\sin \alpha}
$$

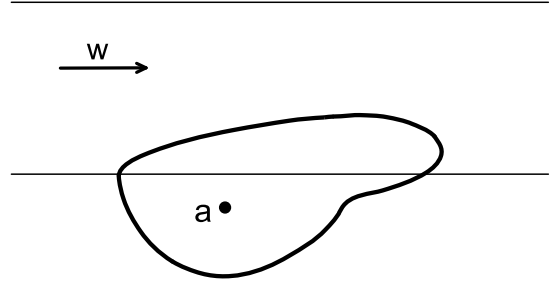

Figure 5. A schematic picture of the one-side "total reflection" of autowaves at the boundary between the excitable media. 
where $w^{*}$ is given by equation (3.4) and has the meaning of the critical velocity for $\alpha=\pi / 2$. The steady-state structure shown in figure $4 \mathrm{~b}$ can be analyzed in a similar fashion.

In closing, we briefly describe an interesting phenomenon which can be observed on a boundary between the moving and fixed excitable media. In a sense this phenomenon may be called the one-side "total reflection" of autowaves. Suppose that a circular autowave was excited at some point "a" in the fixed medium (figure 5). For simplicity, the centre of the wave is assumed to be far removed from the boundary so that the curvature dependence of the front velocity near the boundary can be neglected. When the front reaches the boundary it generates the wave in the moving medium. The sources of this secondary wave are two intersection points of the incident wave and the boundary. One point is to the left, and the other is to the right of a vertical axis $Y$ passing through the centre of the incident wave (figure 5). It is easy to verify that the intersection points move relative the fixed medium with velocity

$$
V_{\mathrm{c}}=V_{0} / \sin \alpha
$$

where $\alpha$ is the angle between the $Y$-axis and the ray traced from the centre "a" to the intersection point. Since the angle $\alpha$ increases with time, the velocity $V_{\mathrm{c}}$ monotonically decreases.

The evolution of a wave front in the moving medium essentially depends on whether its points are to the left or to the right of the $Y$-axis. First we consider the front to the right of the $Y$-axis. Sooner or later, the velocity of the intersection point of the incident wave becomes equal to $V_{0}+w$. As follows from (4.4), it occurs for $\alpha=\alpha_{0}$, where

$$
\sin \alpha_{0}=\frac{V_{0}}{V_{0}+w}
$$

From this time on the right intersection point will not generate the wave in the moving medium. In contrast, a "reflected" wave will be generated in the fixed medium. As a result, the wave front arranges itself into the form shown in figure 5 . It should be noted that the angle between the "reflected" wave and the $Y$-axis at the boundary will not vary with time and will be equal to $\alpha_{0}$ given by equation (4.5).

Clearly the velocity of the left intersection point in figure 5 will never become smaller than the velocity of the wave front in the moving medium. Therefore no "reflected" wave is generated at this point and, as time elapses, the wave front near the boundary takes the shape shown in figure $4 \mathrm{~b}$. The resulting structure is illustrated in figure 5.

By analogy with optics, the above effect may be called the one-side "total reflection" of autowaves at a boundary between moving and fixed excitable media. It is interesting to note that a similar effect was observed at a boundary between excitable media with different diffusion coefficients [14]. 


\section{Computer simulations}

The above considerations were based on the kinematic equation (2.1) and the eikonal equation (2.2), which may be regarded as a phenomenological approach describing the main features of the "microscopic" model of excitable media, equations (1.1). It is of interest to check our analytic results by numerical solution of reaction-diffusion equations.

We have performed computer simulations of equations (1.1) for a two-variable excitable medium [3]:

$$
\frac{\partial u}{\partial t}=F_{u}(u, v)+D_{u} \Delta u, \quad \frac{\partial v}{\partial t}=\varepsilon F_{v}(u, v)+D_{v} \Delta u
$$

where $u$ and $v$ are the activator and inhibitor variables respectively, $D_{u}$ and $D_{v}$ are the diffusion coefficients of both species. The formal parameter $\varepsilon$ determines the time scale for the inhibitor relaxation. We used the model with piecewise linear generic reactivity functions $F_{u}$ and $F_{v}$ (see, e.g., [13]):

$$
F_{u}(u, v)=f(u)+v, \quad F_{v}(u, v)= \begin{cases}k_{v} u-v, & k_{v} u-v \geqslant 0 \\ k_{\varepsilon}\left(k_{v} u-v\right), & k_{v} u-v<0\end{cases}
$$

where

$$
\begin{aligned}
& f(u)= \begin{cases}-k_{1} u, & u<\sigma \\
k_{u}(u-a), & \sigma \leqslant u \leqslant 1-\sigma \\
k_{2}(1-u), & 1-\sigma<u,\end{cases} \\
& k_{1}=\frac{a-\sigma}{\sigma} k_{u}, \quad k_{2}=\frac{1-\sigma-a}{\sigma} k_{u} .
\end{aligned}
$$

Constants $a, \sigma, k_{u}, k_{v}, k_{\varepsilon}$ are free parameters of the model. In all simulations the values of these parameters were

$$
a=0.1, \quad \sigma=0.01, \quad k_{u}=1.7, \quad k_{v}=2, \quad k_{\varepsilon}=6 .
$$

The dimensionless activator diffusion coefficient was $D_{u}=1.5 \cdot 10^{-4}$. Since the main objective of computer simulations was to test the qualitative properties of autowaves discussed in the previous sections, we have restricted ourselves to the simplest model with $D_{v}=0$.

Equations (5.1) were integrated in a 2D array of $128 \times 128$ elements using the Runge-Kutta method with the following boundary conditions:

(i) Zero-flux boundary conditions for the reagents at the top and bottom of the medium;

(ii) Periodic boundary conditions at the left and right boundaries of the domain; 


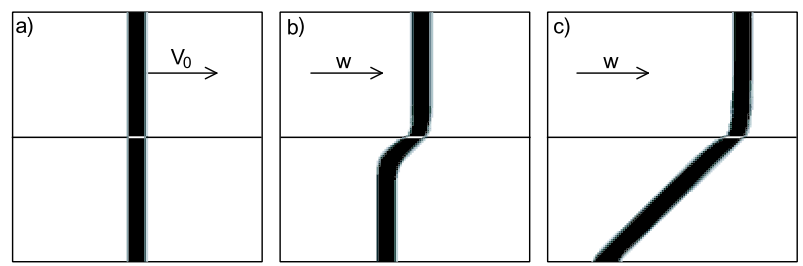

Figure 6. Formation of a stationary wave structure at the boundary between the moving and fixed excitable media. The velocity of the plane front $V_{0}=0.023$, the velocity of the medium $w=0.008$; a) $t=0$, b) $t=18.4$, c) $t=70$.
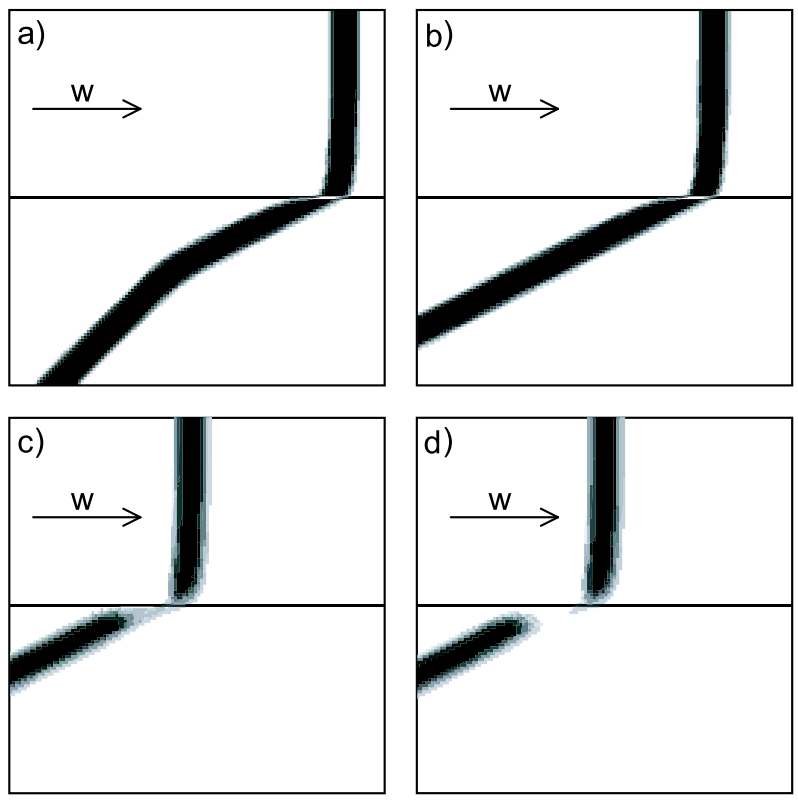

Figure 7. Excitation fronts for different relative velocities of the media: a) The value of $w$ is changed from $w=0.008$ to $w=0.04$ (subcritical behavior); $\mathrm{b}$ ) The stable structure for $w=0.04 ; \mathrm{c}), \mathrm{d}$ ) The value of $w$ is changed from $w=0.04$ to $w=0.05\left(w^{*} \approx 0.045\right)$.

(iii) Continuity conditions for the concentrations $u, v$ and their gradients at the boundary between the moving and fixed media.

Note also that the drift terms $\boldsymbol{w} \cdot \boldsymbol{\nabla} u$ and $\boldsymbol{w} \cdot \boldsymbol{\nabla} v$ must be added in the left-hand sides of equations (5.1) for the moving medium.

The results of computer simulations are shown in figures 6-8. Figure 6 illustrates the formation of a stationary structure at the boundary between the moving and fixed media. Initially the plane excitation front was generated in the homogeneous medium with $w=0$ (figure 6a). Then, at time $t=0$ the upper half of the medium begins to move with velocity $w$. After a lapse of time the stationary structure is formed (figure $6 \mathrm{c}$ ). For $w<w^{*}$, the increase of the relative velocity $w$ of the media leads only to distortions of the structure which move along the $V$-shaped branch in the fixed medium and a new stable wave front is formed (figures $7 \mathrm{a}$ and $7 \mathrm{~b}$ ). If 

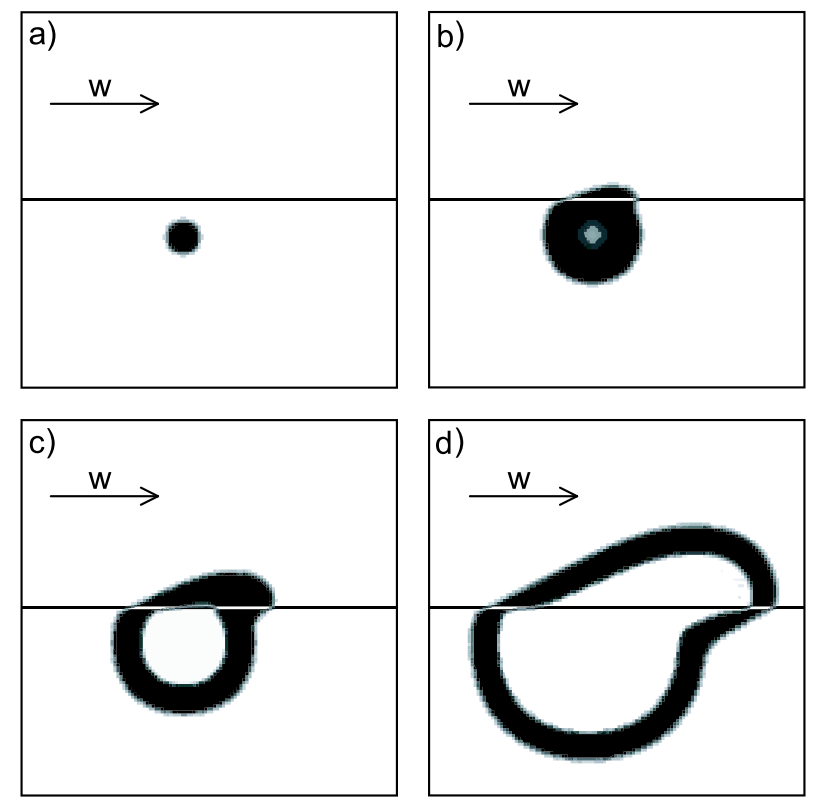

Figure 8. Computer simulation of the "total reflection" of autowaves: a) $t=1.4$; b) $t=5.5$; c) $t=7.9$; d) $t=13.9$. $V_{0}=0.023, w=0.03$.

$w$ exceeds the critical value $w^{*}$, the wave front breaks at the boundary (figures $7 \mathrm{c}$ and $7 \mathrm{~d}$ ) in accordance with the prediction of the kinematic theory.

Finally, the "total reflection" of autowaves is shown in figure 8. The resulting structure coincides with that predicted by the kinematic theory (see figure 5).

\section{Conclusion}

In this paper we have discussed some effects related to autowave dynamics in the moving excitable media. An important point is that the qualitative features of these effects are common to a variety of reaction-diffusion excitable media with different generic reactivity terms $\boldsymbol{F}$ in equations (1.1). Considerable attention has been recently focussed on mesoporous glasses as a support for the BZ reaction $[15,16]$. These "solid" excitable media appear to be best suited for experimental observation of the critical properties and refraction of autowave discussed in section 3 and section 4 .

It should be noted that the method for studying stationary autowave structures proposed in this paper applies to any piecewise homogeneous $2 \mathrm{D}$ excitable media with plane boundaries between the parts having different properties. For instance, it is not difficult to consider the refraction of autowaves at a boundary between excitable media with different diffusion coefficients and velocities of the plane front.

Within the last decade, there has been a marked interest in the study of autowave patterns in fluid media. Recently a simple kinematic model was proposed to explain some features of the spiral wave meandering induced by fluid convection [6], but a methodical kinematic theory of autowaves in reaction-diffusion-convection media is still lacking. We intend to consider this important problem in future publications. 


\section{Acknowledgement}

This work was partially supported by U.S.CRDF-RF Ministry of Education Award VZ-010-0.

\section{References}

1. Mikhailov A.S. Foundations of Synergetics I. Distributed Active Systems. Berlin, Springer, 1990.

2. Klimontovich Yu.L. Statistical Theory of Open Systems. Dordrecht, Kluwer, 1995.

3. Davydov V.A., Zykov V.S., Mikhailov A.S. // Sov. Phys. Usp., 1991, vol. 34, p. 665.

4. Mikhailov A.S., Davydov V.A., Zykov V.S. // Physica D, 1994, vol. 70, p. 1.

5. Pérez-Villar P., Munuzuri A.P., Perez-Munuzuri V. // Phys. Rev. E, 2000, vol. 61, p. 3771.

6. Pérez-Villar P., Munuzuri A.P., Lorenzo M.N., Perez-Munuzuri V. // Phys. Rev. E, 2002, vol. 66, p. 036309.

7. Davydov V.A., Davydov N.V., Stolyarov M.N., Yamaguchi T. Autowave patterns in moving excitable media. - In: Abstracts of International conference "Criteria of selforganization in physical, chemical, and biological systems". Moscow-Suzdal, Russia, 1995, p. 117.

8. Foerster P., Müller S.C., Hess B. // Science, 1988, vol. 241, p. 685.

9. Brazhnik P.K., Davydov V.A. // Phys. lett. A, 1995, vol. 199, p. 40.

10. Brazhnik P.K., Tyson J.J. // Physica D, 1997, vol. 102, p. 300.

11. Pérez-Muñuzuri V. et al. // Physica D, 1996, vol. 94, p. 148.

12. Zykov V.S. // Biophysics, 1980, vol. 25, p. 906.

13. Zykov V.S. Simulations of Wave Processes in Excitable Media. Manchester, Manchester Univ. Press, 1987.

14. Zhabotinsky A.M., Eager M.D., Epstein I.R. // Phys. Rev. Lett., 1993, vol. 71, p. 1526.

15. Amemiya T., Nakaiva M., Ohmori T., Yamaguchi T. // Physica D, 1995, vol. 84, p. 103.

16. Yamaguchi T., Amemiya T., Ohmori T., Morikawa T., Kusumi T., H. Nagasawa T. // Macromolecular Symposia, 2000, vol. 160, p.131. 


\title{
Автохвилі в рухомих збуджених середовищах
}

\author{
В.А.Давидов ${ }^{1}$, М.В.Давидов ${ }^{1}$, В.Г.Морозов ${ }^{1}$, \\ М.Н.Столяров ${ }^{2}$, Т.Ямагучі $^{3}$
}

1 Московський державний інститут радіотехніки, електроніки та автоматики, 117454, проспект Вернадського 78, Москва, Росія

2 Відділення теоретичної фізики Інституту фізики РАН, 117924, Ленінський проспект 53, Москва, Росія

3 Національний інститут сучасної науки та техніки, Хігаші, 1-1-1, Цукуба, Ібаракі 305-8565, Японія

Отримано 5 квітня 2004 р.

В рамках кінематичної теорії автохвиль запропоновано метод для аналітичного опису стаціонарних автохвильових структур, що виникають на межі між рухомим та нерухомим збудженими середовищами. Передбачено явище розриву фронту автохвилі в таких структурах. Розглядається заломлення автохвилі та одностороннє "повне відбиття"на границі. Отримані аналітичні результати знайшли своє підтвердження при комп'ютерному моделюванні. Обговорюються перспективи застосування запропонованого методу для подальшого дослідження автохвильової динаміки в рухомих середовищах, що збуджуються.

Ключові слова: нелінійна динаміка та нелінійні динамічні системи, реакційно-дифузійні середовища, заломлення автохвиль

PACS: $05.50 .+q, 05.70 . \operatorname{Ln}, 82.40 .-g, 87.10 .+e$ 